\title{
Large Hadron Collider momentum calibration and accuracy
}

\author{
E. Todesco and J. Wenninger* \\ CERN, CH-1211 Geneva 23, Switzerland \\ (Received 1 March 2017; published 21 August 2017)
}

\begin{abstract}
As a result of the excellent quality of the Large Hadron Collider (LHC) experimental detectors and the accurate calibration of the luminosity at the LHC, uncertainties on the LHC beam energy may contribute significantly to the measurement errors on certain observables unless the relative uncertainty is well below $1 \%$. Direct measurements of the beam energy using the revolution frequency difference of proton and lead beams combined with the magnetic model errors are used to provide the energy uncertainty of the LHC beams. Above injection energy the relative uncertainty on the beam energy is determined to be $\pm 0.1 \%$. The energy values as reconstructed and distributed online to the LHC experiments do not require any correction above injection energy. At injection a correction of $+0.31 \mathrm{GeV} / \mathrm{c}$ must be applied to the online energy values.
\end{abstract}

DOI: 10.1103/PhysRevAccelBeams.20.081003

\section{INTRODUCTION}

Operation of the Large Hadron Collider (LHC) between 2009 and 2016 saw an impressive progression of the peak and integrated luminosity delivered to the LHC experiments. In total close to $30 \mathrm{fb}^{-1}$ were delivered at $3.5 \mathrm{TeV} / \mathrm{c}$ and $4 \mathrm{TeV} / \mathrm{c}$, while around $45 \mathrm{fb}^{-1}$ were delivered at $6.5 \mathrm{TeV} / \mathrm{c}$. The LHC experiments have measured the integrated luminosity of the different data set with a few percent precision using the Van de Meer scan method [15]. This allows for very precise production cross section measurements of heavy particles such as $W, Z$ bosons and top quark pairs. In this case the uncertainty on the beam energy becomes an important uncertainty when comparing the measured values to the theoretical prediction which is energy dependent. The beam energy uncertainty also plays an important role in elastic scattering measurements carried out by the TOTEM [6] and ATLAS Collaborations [7]. At the time of writing of this document the LHC experiments have expressed the wish to know the LHC beam energy with an accuracy much better than $1 \%$.

The 12 year beam energy calibration program of LEP, formerly installed in the LHC tunnel, was extremely successful in providing accurate beam energy measurements between $40 \mathrm{GeV} / \mathrm{c}$ and $100 \mathrm{GeV} / \mathrm{c}[8,9]$. Although resonant depolarization, the workhorse of LEP energy calibration, is not available at the LHC, the experience gained on LEP is also relevant for LHC energy calibration. Based on the LEP and SPS experience with energy

\footnotetext{
*Jorg.Wenninger@cern.ch

Published by the American Physical Society under the terms of the Creative Commons Attribution 4.0 International license. Further distribution of this work must maintain attribution to the author(s) and the published article's title, journal citation, and DOI.
}

calibration first estimates for the expectations at the LHC were described in a note [10].

This document begins with a brief description of the main ingredients to the energy of a storage ring. The knowledge and the results of the LHC dipole field model is discussed in detail. Effects leading to time dependence of the LHC beam momentum are then presented. A direct calibration technique based on the comparison of proton and ion beams is discussed in detail. Beam momentum measurements at $450 \mathrm{GeV} / \mathrm{c}, 4 \mathrm{TeV} / \mathrm{c}$, and $6.5 \mathrm{TeV} / \mathrm{c}$ are presented together with their measurement errors. Finally the corrections to the energy values as reconstructed and distributed online to the LHC experiments are presented together with their uncertainties.

\section{LHC OPERATION ENERGIES}

Between 2009 and 2016, the main LHC proton-proton (pp) production runs were performed at beam energies of 3.5 TeV/c (2010 and 2011), $4 \mathrm{TeV} / \mathrm{c}$ (2012) and 6.5 TeV/c (2015 and 2016). Lead-lead (PbPb) collisions were delivered at $3.5 \mathrm{TeV} / \mathrm{c}$ (2010 and 2011) and 6.37 TeV/c (2015). Proton-lead (pPb) collisions were delivered at $4 \mathrm{TeV} / \mathrm{c}$ (2013 and 2016) and $6.5 \mathrm{TeV} / \mathrm{c}$ (2016). For lead beams the momenta must be multiplied by the ion charge $Z$, where $Z$ equals 82 because the ions are fully stripped. The lead isotope used at $\mathrm{LHC}$ is $\mathrm{Pb}_{208}$. Special proton reference runs were delivered at $1.38 \mathrm{TeV} / \mathrm{c}$ and $2.51 \mathrm{TeV} / \mathrm{c}$, they correspond to proton center-of-mass energies equivalent to the average nucleon-nucleon centerof-mass energy of the $\mathrm{PbPb}$ runs at $3.5 \mathrm{TeV} / \mathrm{c}$ and $6.37 \mathrm{TeV} / \mathrm{c}$, respectively $\mathrm{pPb}$ runs at $4 \mathrm{TeV} / \mathrm{c}$. Table I presents an overview of the operating energies of the LHC.

\section{BEAM MOMENTUM AND MAGNETIC FIELDS}

In a storage ring like the LHC the beam momentum $P$ of each ring is defined by the integral of the bending field $B$ along the closed orbit of each beam 
TABLE I. LHC operation energies with beam mode and corresponding year.

\begin{tabular}{lcc}
\hline \hline Momentum $(\mathrm{GeV} / \mathrm{c})$ & Beam mode & Year \\
\hline 1380 & $\mathrm{pp}$ & 2011,2013 \\
2510 & $\mathrm{pp}$ & 2015 \\
3500 & $\mathrm{pp} \mathrm{PbPb}$ & 2010,2011 \\
4000 & $\mathrm{pp} \mathrm{pPb}$ & $2012,2013,2016$ \\
6370 & $\mathrm{PbPb}$ & 2015 \\
6500 & $\mathrm{pp} \mathrm{pPb}$ & 20152016 \\
\hline \hline
\end{tabular}

$P=\frac{Z e}{2 \pi} \oint B(s) d s=Z \times 47.7[\mathrm{MeV} / \mathrm{c} / \mathrm{Tm}] \oint B(s) d s$,

where $Z e$ is the particle charge, $Z=1$ for protons and $Z=82$ for $\mathrm{Pb}^{82+}$ lead ions, and $s$ is the longitudinal coordinate along the beam orbit. The contributions of the different magnet multipoles to the beam momentum can be decomposed into 3 main terms,

$$
P=P_{d}+\Delta P_{q}+\Delta P_{\epsilon} .
$$

Here $P_{d}$ is the contribution of the dipoles and $\Delta P_{q}$ is the correction to the energy due the quadrupoles. Other elements, for example horizontal orbit correctors used for beam steering, can give additional small contributions $\Delta P_{\epsilon}$ to the momentum. $P_{d}$ depends on the integrated dipole field $(B L)_{d}$ and accounts usually for almost $100 \%$ of the beam energy since the dipoles define the nominal momentum,

$$
P_{d}=\frac{e}{2 \pi}(B L)_{d} .
$$

The relative energy change $\Delta P_{q} / P$ can be expressed in terms of orbit length $C$ or alternatively rf frequency $f_{\text {rf }}$

$$
\frac{\Delta P_{q}}{P}=-\frac{1}{\eta} \frac{C-C_{c}}{C}=\frac{1}{\eta} \frac{f_{\mathrm{rf}}-f_{\mathrm{rfc}}}{f_{\mathrm{rf}}} .
$$

It is a function of the central orbit length (circumference) $C_{c}$ or central $\mathrm{rf}$ frequency $f_{\mathrm{rfc}}$ and of the slippage factor $\eta=1 / \gamma^{2}-\alpha . \alpha$ is the lattice momentum compaction factor, $\alpha \simeq 3.2 \times 10^{-4}$ for the LHC and $\gamma=E / m$ is the ratio of energy over rest mass. At LHC energies to good approximation $\eta \cong-\alpha$. The central orbit length and central $\mathrm{rf}$ frequency correspond to the orbit where the beam is centered on average in the quadrupoles; on this orbit $\Delta P_{q}$ vanishes. In general $\Delta P_{q} / P$ does not account for more then a per-mill of the bending field integral. For a perfectly aligned machine the definition of the central frequency $f_{\text {rf }}^{c}$ (and of the central orbit length) is unambiguous. It corresponds to the rf frequency (or orbit length) for which the beam is centered in all quadrupoles. In a real machine with misaligned magnets the beam is travelling on a closed orbit that is not centered in each quadrupole. In such a case the central frequency corresponds to the rf frequency for which the beam is centered on average in the quadrupoles.

\section{A. Center of mass energy}

At the interaction point of an LHC experiment each beam is characterized by its relativistic energy-momentum vector $P_{\mu}=(E, \vec{p})$ where $E$ is the beam energy and $\vec{p}$ the momentum vector. The center of mass energy $E_{\mathrm{CM}}$ is defined as

$E_{\mathrm{CM}}^{2}=\left\|P^{(1)}+P^{(2)}\right\|^{2}=\left(E^{(1)}+E^{(2)}\right)^{2}-\left(\vec{p}^{(1)}+\vec{p}^{(2)}\right)^{2}$

where $(i)$ labels the two beams. For two beams with identical energies $E^{(1)}=E^{(2)}=E$ colliding head-on, $\vec{p}^{(1)}+\vec{p}^{(2)}=0, E_{\mathrm{CM}}$ is just the sum of the two beam energies, $E_{\mathrm{CM}}=E^{(1)}+E^{(2)}=2 E$.

\section{LHC MAIN MAGNET FIELD}

\section{A. Field accuracy based on construction tolerances}

\section{The Biot-Savart contribution}

We will first discuss the relation between magnetic field accuracy, magnet design, and construction tolerances. The LHC dipoles are electromagnets where the main component of the magnetic field is proportional to the magnet current [11]. The magnet cross section, and the detail of the coil are shown in Fig. 1. The radius of the magnet aperture is $28 \mathrm{~mm}$, and the coil is made with two layers of a 15.4-mm-width superconducting cable. There are 15 turns in the inner layer and 25 turns in the outer layer. The cables have different cross sections, but carry the same current, that is $11.85 \mathrm{kA}$ at nominal field for a total of $474 \mathrm{kA}$-turns. The nominal field in the bore is $8.3 \mathrm{~T}$; most of it is given by the current lines, plus a second order effect $(\sim 18 \%)$ given by iron, placed at $98 \mathrm{~mm}$ from the centre of the aperture. The magnetic field is given by the product of the current times the $1 / r$ term of the Biot-Savart law. The current is known with an accuracy of a few parts per million $[12,13]$. The uncertainty on the magnetic field is therefore driven by the precision of the positioning of the current lines.

We first consider a very simplified model, in which the coil is lumped in four current lines at $30^{\circ}$, as in a Helmholtz coillike configuration (see Fig. 2 top). The field will be given by

$$
B=4 \frac{\mu_{0} I_{H}}{2 \pi r_{H}} \sin \frac{\pi}{3}
$$

where the current intensity $I_{H}$ is the total number of ampereturns and for the current line position we can use the average of $1 / r$ 

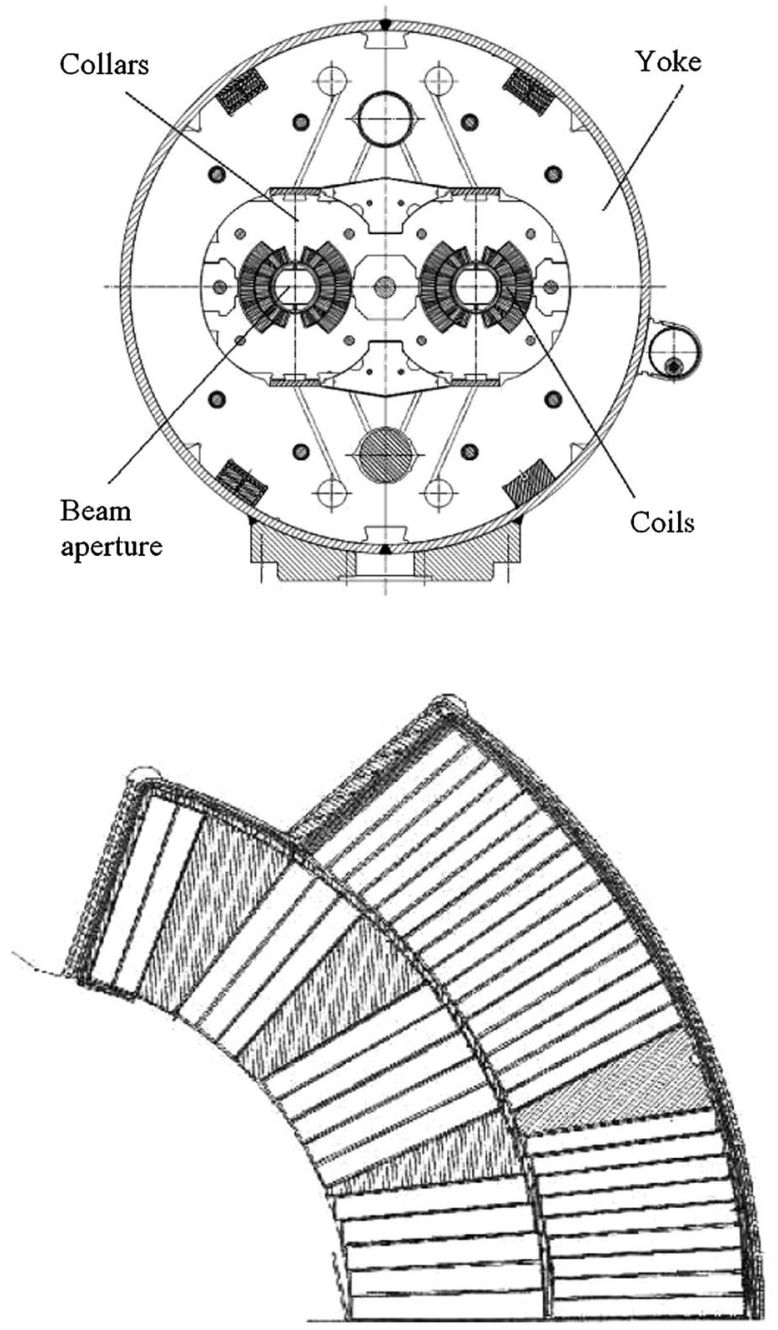

FIG. 1. LHC dipole cross-section (top) and detail of one quarter of the coil (bottom).

$$
\frac{1}{r_{H}}=\frac{1}{2}\left(\frac{1}{r}+\frac{1}{r+w}\right)=\frac{1}{2} \frac{w+2 r}{r(w+r)}
$$

where $r=28 \mathrm{~mm}$ is the magnet aperture radius, and $w=31 \mathrm{~mm}$ the width of the layer. The above average gives $r_{H}=38 \mathrm{~mm}$, and with this very rough model one already obtains $B=8.7 \mathrm{~T}$, not far from the $8.3 \mathrm{~T}$ value. The sensitivity of the magnetic field accuracy, given by the positioning of the current line, is

$$
\frac{\Delta B}{B}=\frac{\Delta r_{H}}{r_{H}}
$$

Since the tolerances for the current line positioning are $\pm 0.05 \mathrm{~mm}$, the relative accuracy on $B$ is $\pm 0.13 \%$. The contribution to the uncertainty of the angle is a second order effect with respect to the radial part. This simple estimate proves that the order of magnitude for the field accuracy due to construction tolerances is around $0.1 \%$. To improve the
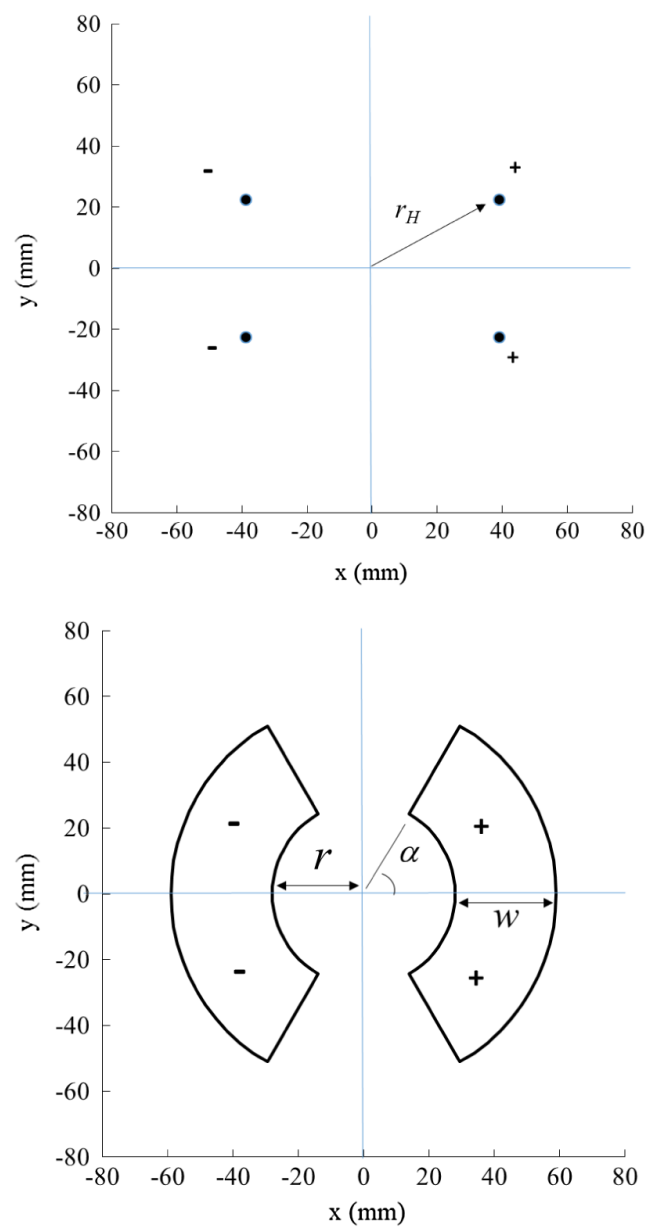

FIG. 2. A magnet given by a Helmholtz like coil (top) and a sector coil (bottom).

field accuracy by an order of magnitude, i.e. to the level of $0.01 \%$, the current lines should be positioned with tolerances of around $5 \mu \mathrm{m}$, which is well beyond the state of the art for coils made with Rutherford cables.

We now consider a more realistic model (see Fig. 2 bottom) where the coil is a circular sector of width $w$, piling up $N$ trapezoidal cables with mid thickness $t$. The coil has a pole angle given by

$$
\alpha=\frac{N t}{r+w / 2},
$$

and the field is given by

$$
B=\frac{2 \mu_{0}}{\pi} j w \sin \alpha
$$

where $j$ is the current density in the cable. The above expression can be written as 


$$
\begin{aligned}
B & =\frac{2 \mu_{0}}{\pi} j w \frac{N t}{(r+w / 2)} \frac{\sin \alpha}{\alpha} \\
& =\frac{2 \mu_{0} I N}{\pi} \frac{1}{r+w / 2} \frac{\sin \alpha}{\alpha} .
\end{aligned}
$$

As we already pointed out, the ampere-turns give a negligible contribution to the field accuracy since the current is known within ppm and the number of turns is an integer. The radial positioning uncertainty contribution to the field error is $\pm 0.05 /(28+15.4)= \pm 0.12 \%$, similar to the previous model. The coil pole angle is placed with an accuracy of $0.05 \mathrm{~mm}$, corresponding to a $0.12 \%$ accuracy in $\alpha$, and $0.05 \%$ on $\sin (\alpha) / \alpha$. A quadratic sum of the contributions yields a final error estimate of $0.13 \%$, similar to the Helmholtz-like coil model.

In the LHC dipoles, the iron is placed at $R_{I}=98 \mathrm{~mm}$ distance from the center, over the whole circumference excluding about $\pm 45^{\circ}$ in the direction of the magnet center (see Fig. 1 top). An analytical estimate for the main field increase due to a circular iron using the image currents is given by

$$
\zeta=\frac{\Delta B}{B}=\frac{r(r+w)}{R_{I}^{2}},
$$

and for the LHC dipole coil one obtains a main field increase of $17 \%$. From this approximate expression it is possible to estimate the uncertainty on this quantity due to the geometrical tolerances

$$
\begin{aligned}
\frac{\Delta \zeta}{\zeta} & =\frac{\Delta r}{r}+\frac{\Delta(r+w)}{r+w}+2 \frac{\Delta R_{I}}{R_{I}} \\
& =\frac{0.05}{28}+\frac{0.05}{59}+2 \frac{0.1}{98} \simeq 0.5 \%
\end{aligned}
$$

giving an error on the main field of the order of $0.08 \%$, below the errors related to the coil positioning. The magnetic model accuracy may be improved by accounting for the actual shape of the iron, for the wedges used to optimize the dipole field quality (there are four wedges in the LHC dipole coils, see Fig. 1), and for the trapezoidal geometry of the cable. For such a refined model the impact of the construction tolerances on the field accuracy may be estimated with a Monte Carlo simulation. The results indicate once more an intrinsic accuracy of the BiotSavart contribution of $0.13 \%$.

\section{Nonlinear contributions}

The previous estimates of the field accuracy are valid in the momentum range between 2 and $5 \mathrm{TeV} / \mathrm{c}$, where the magnetic field is proportional to the current within $0.01 \%$. At lower and at higher fields two nonlinear effects whose modeling is much more complex than Biot-Savart law contribute to the magnetic field.
At $7 \mathrm{TeV} / \mathrm{c}$ the main field is $8.33 \mathrm{~T}$ and close to the coil the iron is well above the saturation field of $2 \mathrm{~T}$. The iron therefore becomes a nonlinear element: it stops acting like a mirror for the current lines and it progressively becomes transparent for higher fields. This corresponds to a decrease of the ratio main field/current (transfer function) of $0.78 \%$ according a finite element model.

At $450 \mathrm{GeV} / \mathrm{c}$ the magnetic field is affected by magnetization currents that shield the superconductor against the change of field during the field ramp. Whereas the impact on high order multipoles (like the sextupolar component) is relatively large [14], for the main component the effect is within $0.1 \%$ [15].

In summary two nonlinear contributions affect injection and high energy: at high energy the contributions are relevant (6 times larger than the field accuracy due to the linear contribution), whereas at injection energy their contribution is similar to the field accuracy due to the linear contribution.

\section{B. Field accuracy based on magnetic measurements}

Magnetic measurements of the LHC main dipoles have been performed using the rotating coils method $[11,16]$. The precision of this method for the main field is $0.01 \%$. Rotating coils are calibrated using reference magnets to reach an accuracy of $0.1 \%$. A cross-check of the calibration method can be based on measuring the same magnets with different coils, or with rotating coils and stretched wires. An example of such a cross-check is shown in Fig. 3.

The LHC dipoles have been measured in different assembly stages and operational conditions [17]: (i) All the dipoles have been measured at room temperature after the collaring (coils in the collars); (ii) All the dipoles have been measured at room temperature after the cold mass

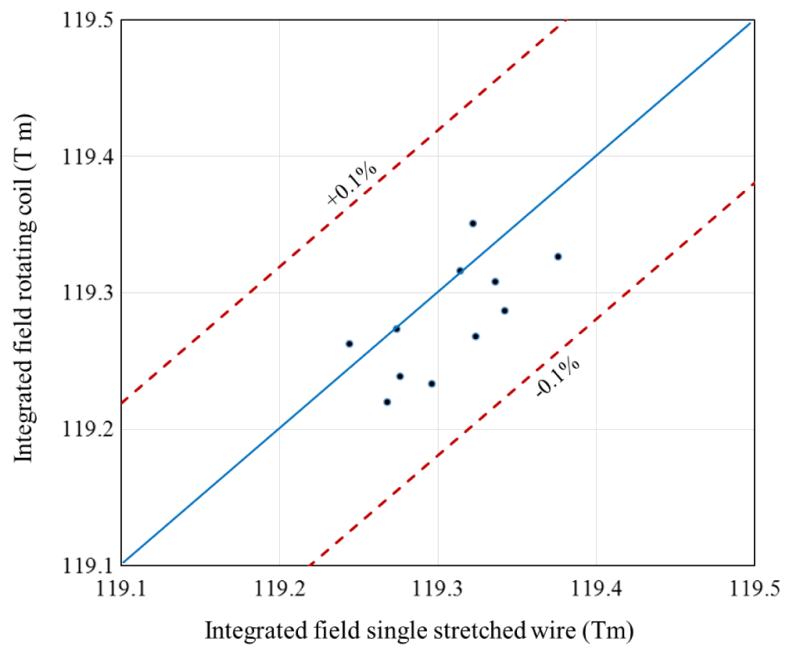

FIG. 3. Comparison between integrated dipole fields measured with single stretched wire and with a rotating coil for $11 \mathrm{LHC}$ dipoles (courtesy of L. Bottura and M. Buzio). The dashed lines correspond to deviations of $\pm 0.1 \%$. 


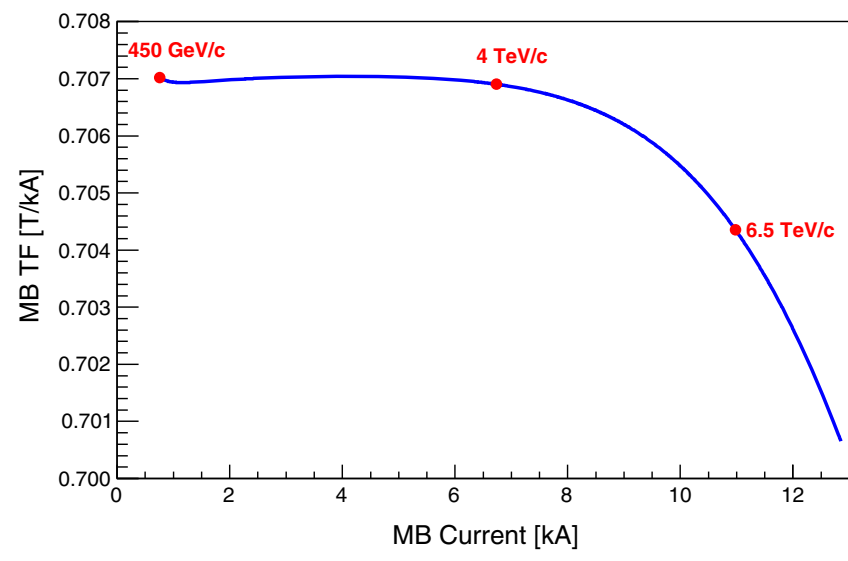

FIG. 4. Transfer function of the LHC main dipole magnets as a function of the circuit current.

assembly (collared coil in the iron yoke with the stainless steel shell, i.e., what is shown in Fig. 1 top); (iii) 200 out of a total of 1232 dipoles have been measured in operational conditions at $1.9 \mathrm{~K}$, with a cycle as in the LHC, over the entire range of operational currents from injection $(0.53 \mathrm{~T})$ to high field $(8.33 \mathrm{~T})$. The resulting field transfer function is presented in Fig. 4. The magnetic cycle used for the measurements was identical to the cycle used operationally at the LHC to ensure that the magnet remain on the same ascending hysteresis branch.

The transfer functions used in the LHC control system to convert a desired beam momentum into magnet currents is based on the geometric value measured on each individual magnet at room temperature, plus the offset due to the nonlinear effects averaged on the sample of measured magnets at $1.9 \mathrm{~K}$.

\section{A cross-check between model and measurements}

In order to further validate our analysis we present the agreement between model and magnetic measurements. The LHC dipoles were constructed with three successive iterations of the coil geometry. Here we recall the results relative to the comparison of a model [18] using the detailed layout of the superconducting strands to the measurements of the so called geometric component of the transfer function (TF) [19]. The geometric TF is defined as the value of the magnetic field divided by the current at $5 \mathrm{kA}$ where nonlinear effects are not present. This corresponds to a momentum of $2.9 \mathrm{TeV} / \mathrm{c}$. The difference between model and measurements for the three iterations of the coil geometry is within $0.1 \%$, see Table II. This is in agreement with the expected accuracy of the measuring system and of the magnet modeling.

The nonlinear component due to iron saturation has a larger discrepancy between model and measurement: at $7 \mathrm{TeV}$ we expect a reduction of the ratio field/current of $0.78 \%$ from the model, whereas the measurements yield only $0.62 \%$ [19]. This discrepancy is due to a limited
TABLE II. Measured and modeled transfer function (TF) for the three LHC dipole cross section types.

\begin{tabular}{lccc}
\hline \hline $\begin{array}{l}\text { Cross-section } \\
\text { type }\end{array}$ & $\begin{array}{c}\text { Measured } \\
\text { TF (T/kA) }\end{array}$ & $\begin{array}{c}\text { Model TF } \\
\text { (T/kA) }\end{array}$ & Difference \\
\hline 1 & 0.70798 & 0.70743 & $-0.08 \%$ \\
2 & 0.70759 & 0.70734 & $-0.04 \%$ \\
3 & 0.70720 & 0.70735 & $0.02 \%$ \\
\hline \hline
\end{tabular}

knowledge of the B-H curve of the iron. The magnetization component affecting the ratio field/current at injection energy is expected to be $-0.05 \%$ from modeling, and the measurement gives an increase of $0.01 \%$ (see Fig. 4).

Summarizing, the estimates of the field based on the Biot-Savart contribution (linear regime of the magnet between 2 and $5 \mathrm{TeV} / \mathrm{c}$ ) confirm the estimates of the field from the magnetic measurements within the accuracies associated to each method. At $7 \mathrm{TeV} / \mathrm{c}$, the saturation component has a model accuracy of the order of $0.2 \%$, whereas at injection the accuracy is better than $0.1 \%$. In all cases magnetic measurements have a higher accuracy than the models.

\section{Magnetic length}

Since for the energy of the particles what counts is not the field in the transverse plane but the integrated field along the magnet, the accuracy of the active length of the magnet - called magnetic length — must be estimated. The LHC dipoles have a magnetic length of $14.36 \mathrm{~m}$ at room temperature and $14.31 \mathrm{~m}$ at $1.9 \mathrm{~K}$. The change corresponds to the thermal contraction of the active part of the magnet. The construction tolerances are of the order of few $\mathrm{mm}$, so well below $0.1 \%$. The measurement precision is also of the order of few $\mathrm{mm}$, so the magnetic length gives a negligible additional contribution to the accuracy of the integrated field.

\section{E. Conclusion on magnetic modeling}

Summarizing, the accuracy on the integrated field in the LHC dipole is $\pm 0.1 \%$ over the entire energy range from $450 \mathrm{GeV} / \mathrm{c}$ to $7 \mathrm{TeV} / \mathrm{c}$. This value is obtained from magnetic measurements. Today the measurements at room temperature and the sampling at $1.9 \mathrm{~K}$ are used in the LHC control system to set the LHC currents at each energy step. Construction tolerances give a field accuracy in the range 2-5 TeV/c equivalent energy of $\pm 0.13 \%$. The comparison between model and measurements confirms both field estimates with the associated accuracies.

An additional cross-check of the accuracy of the magnetic model can be obtained from an analysis of the transverse tunes in the vertical and the horizontal planes. The bare tunes correspond to the tune values obtained by deconvoluting all corrections applied to bring the tunes to their nominal values. Figure 5 presents the evolution of the 


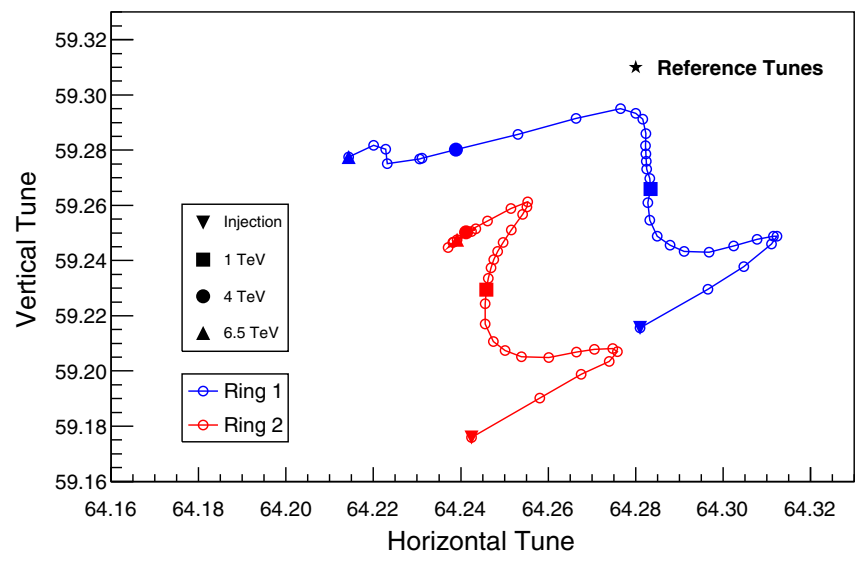

FIG. 5. Evolution of the bare machine tunes (before any correction) along the cycle from injection to $6.5 \mathrm{TeV} / \mathrm{c}$ at constant optics.

bare tunes between injection and $6.5 \mathrm{TeV} / \mathrm{c}$. The maximum tune deviation is $\simeq 0.14$ at injection and $\simeq 0.07$ at $6.5 \mathrm{TeV} / \mathrm{c}$. This corresponds to relative errors of $0.24 \%$ at injection and $0.12 \%$ at $6.5 \mathrm{TeV} / \mathrm{c}$ which agrees well with the expectations for both dipole and quadrupole field error for the LHC.

\section{TIME DEPENDENCE OF THE ENERGY}

The energy of the LHC beams may vary with time due to changes of the dipole field, changes of the orbit corrector settings and geological effects that influence the circumference.

\section{A. Power converters}

The LHC main dipole power converters are regularly calibrated against a precision current source. The current error does not exceed 10 parts per million over a time span a few months between calibrations [13].

\section{B. Orbit corrector magnets}

The horizontal orbit corrector magnets are used primarily to correct the closed orbit of the two beams, but when they are shifted systematically in one direction, their combined field can affect the beam energy [9]. At the LHC the horizontal correctors are used at injection to match the energy of each ring with the energy of the beam extracted from the SPS. Typical corrections are at the level of $\pm 0.03 \%$ and the differences between the rings never exceed $0.02 \%$. This correction is only applied at injection and not propagated to high energy. The energy correction at injection is readjusted typically twice per year using the horizontal orbit correctors. At high energy the relative correction due to correctors does not vary by more than $\pm 0.01 \%$.

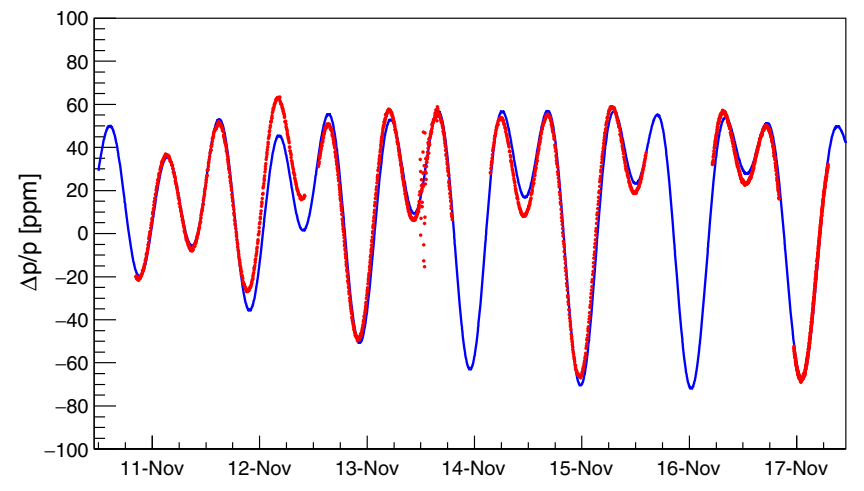

FIG. 6. Predicted (blue line) and observed (red point) tidal energy variations of the LHC ring in November 2016 during long consecutive fills at $4 \mathrm{TeV} / \mathrm{c}$ spanning almost an entire week. The outliers that can be observed around midday November 13th are radial oscillations of the ring induced by the surface waves from a magnitude 7.8 Earthquake in New-Zealand.

\section{Tides and geology}

The circumference of the LHC is oscillating periodically due to Earth tides [20]. The peak-to-peak relative energy swing due to tides reaches $0.014 \%$ which corresponds to a rf frequency swing (to maintain the beam in the center) of $17 \mathrm{~Hz}$. The associated circumference swing is $1.1 \mathrm{~mm}$. An example is given in Fig. 6 for a one week time interval of the 2016 LHC run. The prediction is obtained from former LEP measurements [21,22].

A radial feedback loop corrects the tidal effects by adapting the rf frequency to maintain the beams centered on average in the beam position monitors. This loop is used during ramp and squeeze, it ensures that at the start of the stable colliding beam periods the effect of the tides is essentially zeroed. Tidal effects that occur during the colliding beam periods are compensated by the LHC shift crews or by the radial feedback. Tidal energy shifts, besides being rather small, also tend to average out over the duration of a run.

In addition to periodic tides the ring is also subject to slower seasonal circumference changes that were already observed while LEP was still installed in the LHC tunnel $[9,21,22]$. At the LHC the radial feedback in use during operation compensates automatically such changes. Figure 7 displays the rf frequency adjustments that were made by the radial feedback to maintain the proton beams centered. The data of Fig. 7 has been corrected for the tidal effects. The total frequency swing due to slow geological effects is $35 \mathrm{~Hz}$, which corresponds to a circumference change of $2 \mathrm{~mm}$. If the beam position would not be adjusted by the feedback, the associated relative energy variation would be $0.026 \%$.

In summary the contributions of energy variations over the year and other smaller corrections to the beam energy remain very small, a value of $0.03 \%$ can be considered as a conservative upper limit for the relative energy changes 


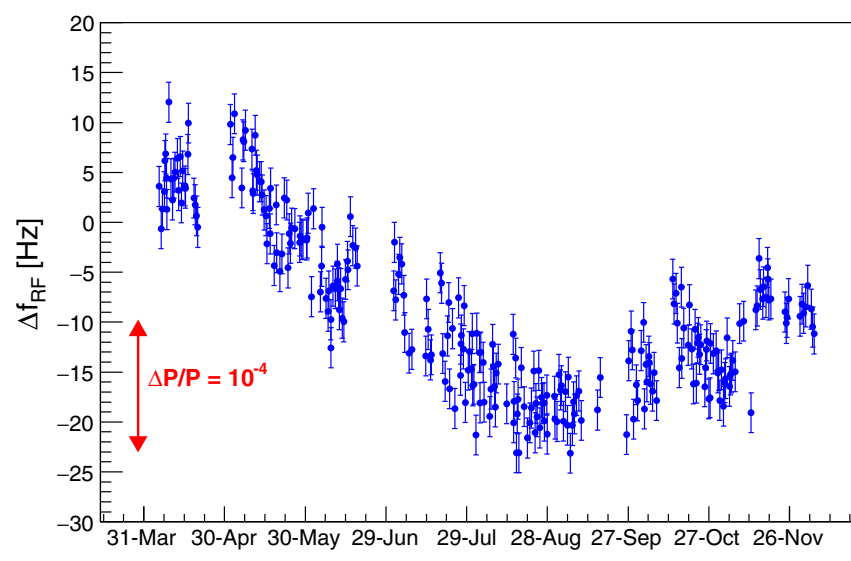

FIG. 7. rf frequency changes required to center the beam in the BPMs at $4 \mathrm{TeV} / \mathrm{c}$ along the $2012 \mathrm{LHC}$ run. The full range corresponds to a circumference change of $2 \mathrm{~mm}$.

over a run above injection energy. Due to the corrections of the radial feedback and the averaging of the tidal effect over time, we associate a relative systematic error of $0.005 \%$ on the energy due to residual geological effects.

\section{Machine reproducibility}

The stability of the LHC dipole field at high energy can only be assessed indirectly since no continuous measurement is available. The reproducibility of the LHC machine transverse tunes over time during the pp run is at the level of $\delta Q \approx \pm 0.002$. For integer tunes of 64 (horizontal plane) and 59 (vertical plane), this yields a dipole field stability of better than $0.003 \%$ for a natural (uncorrected) chromaticity of $\approx-90$ in both planes. Such a good stability at high field is not surprising since the magnets are operated by definition at a stable temperature of $1.9 \mathrm{~K}$, and are cycled in a systematic way before each injection to ensure the highest possible machine reproducibility [23].

\section{ENERGY DIFFERENCE BETWEEN RINGS AND CENTRE-OF-MASS ENERGY}

The main contributions to possible energy differences between the 2 rings are (i) differences in the integrated dipole field along the path of the beams, (ii) differences in the average beam position in the quadrupoles, (iii) differences in the horizontal orbit corrector settings.

The differences in integrated dipole field expected from the magnet measurements are smaller than $0.01 \%$. Such a difference may be estimated with beam data from the corrections that have to be applied to the trim quadrupoles to set the transverse tunes to their nominal values as presented in Fig. 5. While the observed difference is at the level of $0.01 \%$, this method only provides a rough estimate since differences in gradient errors of the quadrupoles between the two rings are also a source of tune trim differences. The two other sources of energy differences (radial position and correctors) may also affect the tune trims, but with a different sensitivity (natural versus total chromaticity). There are no indications that contradict the estimate of $0.01 \%$ for the relative energy difference obtained from the magnet measurements.

Taking into account the observed systematic differences between ring 1 and ring 2, and the very small offsets in the radial position of the two proton beams in pp operation, the maximum difference in average radius is estimated to $\pm 3 \mathrm{~Hz}$ of $\mathrm{rf}$ frequency change. This corresponds to a relative energy difference of $\pm 0.002 \%$.

Energy offsets due to the orbit correctors can be determined directly from the settings of the orbit correctors. Above injection energy a conservative upper limit for the difference is $0.01 \%$.

In summary, adding together all contributions, an upper limit for the relative energy difference between the 2 rings can be set to $\pm 0.02 \%$.

At LEP the center-of-mass energy could differ significantly from one IP to the other due to the large synchrotron radiation loss and the unequal distribution of rf voltage around the machine circumference. This effect is negligible at the LHC due to the very small energy loss of only $6.7 \mathrm{KeV}$ per turn even at $7 \mathrm{TeV} / \mathrm{c}$, the energy loss being proportional to the fourth power of the beam energy.

Given that the energy difference between the two rings is very small compared to the measurement uncertainties and that there are no local energy shifts at the IPs, the centre-ofmass energy equals twice the beam energy. The relative errors on center-of-mass and beam energy are identical because the energy uncertainties of the two rings are highly correlated.

\section{DIRECT BEAM ENERGY MEASUREMENTS}

No measurement device in the form of a reference magnet with magnetic probes or a spectrometer system like at LEP is available at the LHC to determine the energy of the machine during operation. It is however possible to use a technique based on the comparison of $\mathrm{rf}$ frequency of two particle species injected into the same magnetic cycle. This technique was first used for precise energy calibration at LEP with protons and positrons at $20 \mathrm{GeV} / \mathrm{c}$ [24]. Such calibrations were performed at the SPS in 1991 using proton and oxygen ions at $270 \mathrm{GeV} / \mathrm{c}$ [25] and in 2002 using proton and partially stripped $\mathrm{Pb}^{53+}$ lead ions at $450 \mathrm{GeV} / \mathrm{c}[26]$.

\section{A. Rf frequency of protons and ions}

The revolution frequency (respectively the speed) differs for lead ions and protons circulating in the LHC due to the different ratio of charge over rest mass. Since frequencies can be measured with excellent accuracy, this difference between protons and ions can be used to determine the momentum of the beams. 
The speed $\beta c$ of a particle is related to the revolution frequency $f_{\text {rev }}$ and the $\mathrm{rf}$ frequency $f_{\text {rf }}$ by

$$
\beta c=C f_{\text {rev }}=\frac{C f_{\text {rf }}}{h}
$$

where $h$ is the harmonic number of the rf system ( $h=35640$ for the LHC). $C$ is the machine circumference. To determine the speed $\beta$ and therefore the particle momentum, both the machine circumference and the revolution (or rf) frequency must be known.

The momentum and machine circumference may be determined simultaneously by measuring the revolution frequency for two particles with different charge over mass ratio provided that they are injected into exactly the same magnetic machine and on the same orbits. The speed $\beta_{p} c$ of the proton beam is related to its momentum $P$ and its rest mass $m_{p}$ by the well-known relation

$$
\beta_{p}^{2}=\frac{P^{2}}{P^{2}+\left(m_{p} c\right)^{2}}
$$

An ion with charge $Z e$, injected into the same magnetic machine and on the same orbit than the proton beam has a momentum $P_{i}=Z P$. The speed $\beta_{i} c$ of the ions is

$$
\beta_{i}^{2}=\frac{P^{2}}{P^{2}+\left(m_{i} c / Z\right)^{2}}
$$

with $m_{i}$ the ion rest mass. The proton beam momentum $P$ may be expressed in terms of the proton and ion parameters as

$$
P=m_{p} c \sqrt{\frac{\kappa^{2} \mu^{2}-1}{1-\kappa^{2}}}
$$

with

$$
\kappa=\beta_{i} / \beta_{p}=f_{\mathrm{rf}}^{i} / f_{\mathrm{rf}}^{p}
$$

and

$$
\mu=\frac{m_{i}}{Z m_{p}} .
$$

For the fully stripped lead ions with $Z=82$ and atomic mass 208 used at the LHC the value of $\mu$ is 2.517 which reflects the mass over charge ratio of the lead ions. At high energy Eq. (17) can be approximated by

$$
P \cong m_{p} c \sqrt{\frac{f_{\mathrm{rf}}^{p}}{2\left|\Delta f_{\mathrm{rf}}\right|}\left(\mu^{2}-1\right)}
$$

where $\Delta f_{\mathrm{rf}}=f_{\mathrm{rf}}^{p}-f_{\mathrm{rf}}^{i}$ is the $\mathrm{rf}$ frequency difference between the proton and ion beams. The measurement error

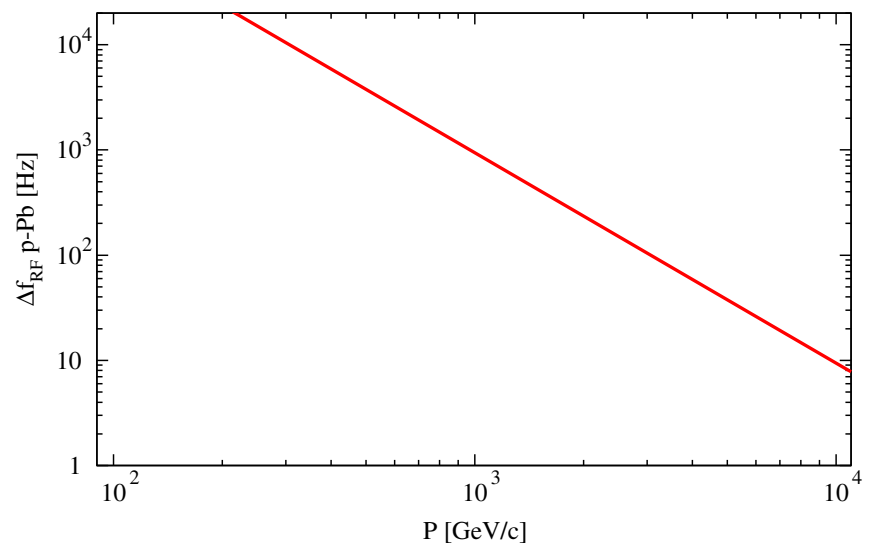

FIG. 8. Expected central rf frequency difference between proton and $\mathrm{Pb}^{82+}$ beams as a function of the beam momentum at the LHC.

on $P$ is dominated by the accuracy of the $\mathrm{rf}$ frequency determination since all other parameters entering Eq. (17) are known with high accuracy.

The frequency difference $\Delta f_{\text {rf }}$ between the beams follows from Eq. (20)

$$
\Delta f_{\mathrm{rf}} \cong\left(\frac{m_{p} c}{P}\right)^{2} \frac{f_{\mathrm{rf}}^{p}}{2}\left(\mu^{2}-1\right)
$$

and it scales quadratically with $\mu$. The dependence on $1 / P^{2}$ makes the measurement increasingly difficult as the momentum increases since the speed differences vanish.

The frequency difference $\Delta f_{\text {rf }}$ is shown as a function of the LHC proton momentum $P$ in Fig. 8, it shrinks by more than 2 orders of magnitude between $450 \mathrm{GeV} / \mathrm{c}$ and $7 \mathrm{TeV} / \mathrm{c}$. Frequency difference values are presented for selected LHC beam energies in Table III.

An accurate calibration at the level of $0.1-1 \%$ in the range of 3.5-7 TeV/c requires a measurement of the radial offset between proton and lead beams at the level of $1-10 \mu \mathrm{m}$ (Table III). This is a very challenging requirement since the LHC ring is not stable to this level on the time scale of a few hours as discussed in the previous section. Due to the limited accuracy of the tidal prediction (at the

TABLE III. This table presents a list of variables that are of interest for a few relevant proton momentum values $(P$, left column). The second column from the left corresponds to the rf frequency difference $\Delta f_{\text {rf }}$ between a proton and ${\mathrm{a} \mathrm{Pb}^{82}+}_{\text {beam. }}$ The third and fourth columns indicate the accuracy $\sigma_{\Delta f}$ on $\Delta f_{\text {rf }}$ and $\sigma_{R}$ on the mean machine radius required to achieve a $0.1 \%$ accuracy for the energy measurement.

\begin{tabular}{lccc}
\hline \hline Momentum $P(\mathrm{GeV} / \mathrm{c})$ & $\Delta f_{\mathrm{rf}}(\mathrm{Hz})$ & $\sigma_{\Delta f}(\mathrm{~Hz})$ & $\sigma_{R}(\mu \mathrm{m})$ \\
\hline 450 & 4650 & 9.1 & 96 \\
4000 & 58.85 & 0.12 & 1.3 \\
6500 & 22.99 & 0.046 & 0.49 \\
7000 & 19.22 & 0.039 & 0.41 \\
\hline \hline
\end{tabular}


level of a few percent) and to the presence of other slow ground movements, it is not possible to accurately predict $C_{c}$ variations to better than $\approx 50 \mu \mathrm{m}$ from one fill to another with a time span of few hours between two fills. A direct measurement of the radial position of the beams is necessary, requiring well calibrated beam position monitors (BPMs) or more complicated measurement techniques [22].

It is challenging to perform accurate measurements as long as the proton and lead beams are injected and ramped in separate machine cycles as it was done up to 2011, with either $\mathrm{pp}$ or $\mathrm{PbPb}$ runs. The situation changed significantly in 2013 and 2016 with the mixed proton-lead runs, where both particle types are present at the same time in the LHC, albeit in separate rings. The frequency offsets can be measured at the same time for protons and ions, canceling out effects from geological deformations of the tunnel and from certain BPM errors.

\section{B. Proton lead beam operation}

Mixed operation mode with protons circulating in LHC ring 1 and lead beams in LHC ring 2 (and vice-versa) was first demonstrated in 2011 [27]. First collisions were delivered in this mode at $4 \mathrm{TeV} / \mathrm{c}$ in September 2012 [28]. A four week long run took place in January 2013, the data used for the beam energy measurement at $4 \mathrm{TeV} / \mathrm{c}$ was collected parasitically during this period. Another run at $6.5 \mathrm{TeV} / \mathrm{c}$ took place in November 2016. Roughly one half of each run was operated with protons in ring 1 and lead in ring 2 , and the other half in the reverse configuration with protons in ring 2 and lead in ring 1.

Operation mixing proton and ions requires special if manipulations. The evolution of the rf frequency in the ramp and on the high energy flat top is shown in Fig. 9. During injection and ramp, the rf systems of the two beams are de-coupled, each beam is operated at the frequency corresponding to a centered beam orbit. The rf frequencies follow the momentum increase during the ramp. On the high energy flat top the rf frequencies of the two beams are forced to a common frequency (typically the average

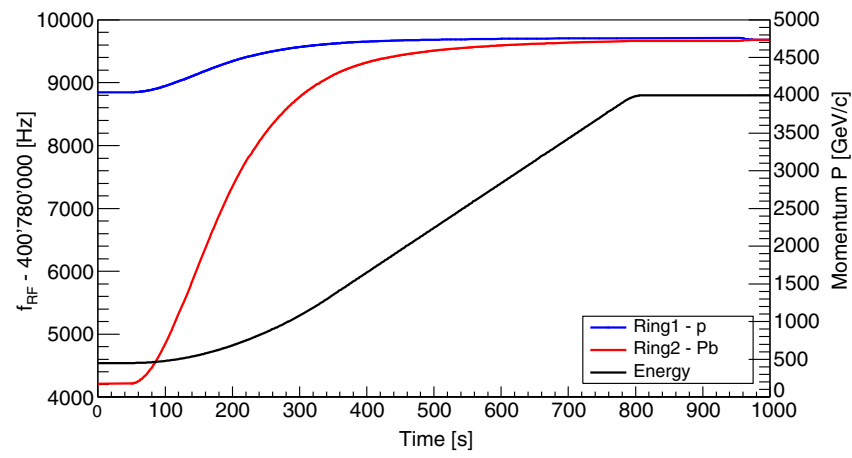

FIG. 9. Evolution of the proton and lead ion $\mathrm{rf}$ frequencies during an energy ramp to $4 \mathrm{TeV} / \mathrm{c}$. The frequencies are locked together at $4 \mathrm{TeV} / \mathrm{c}$ (around time 960). frequency of protons and lead ions), and the beams are resynchronized to ensure that the bunches collide at the experimental interaction points. After the rf manipulations, the two beams move off-center radially since the common frequency does not match the frequency required to center the beams in the quadrupoles. The beams are brought into collision in the experimental interaction regions to deliver collisions for the experiments under those conditions. As a consequence of the off-center orbits, the energy of the proton and lead beams differs by $0.046 \%$ at $4 \mathrm{TeV} / \mathrm{c}$ due to the frequency difference of around $59 \mathrm{~Hz}$. At $6.5 \mathrm{TeV} / \mathrm{c}$ the energy difference is $0.018 \%$. The momentum of the proton beam is always higher than the momentum of the lead beam. The center of mass energy is however not affected because of the energy change is antisymmetric for the two beams.

\section{Frequency offset measurements}

The determination of the beam energy is based on the radial offset of the orbits at the end of the rf manipulations at $4 \mathrm{TeV} / \mathrm{c}$ and $6.5 \mathrm{TeV} / \mathrm{c}$ (phase $3 \mathrm{of} \mathrm{Sec.} \mathrm{VII} \mathrm{B).} \mathrm{The}$ beams are forced on a common frequency that is on average 29(11) $\mathrm{Hz}$ too low for the protons and 29(11) Hz too high for the lead ions at 4(6.5) TeV/c. The proton beam moves radially outward while the lead beam moves radially inward. To determine the beam energy, the radial offsets measured by the 520 dual plane BPMs of each ring are converted into equivalent rf frequency shifts. Figure 10
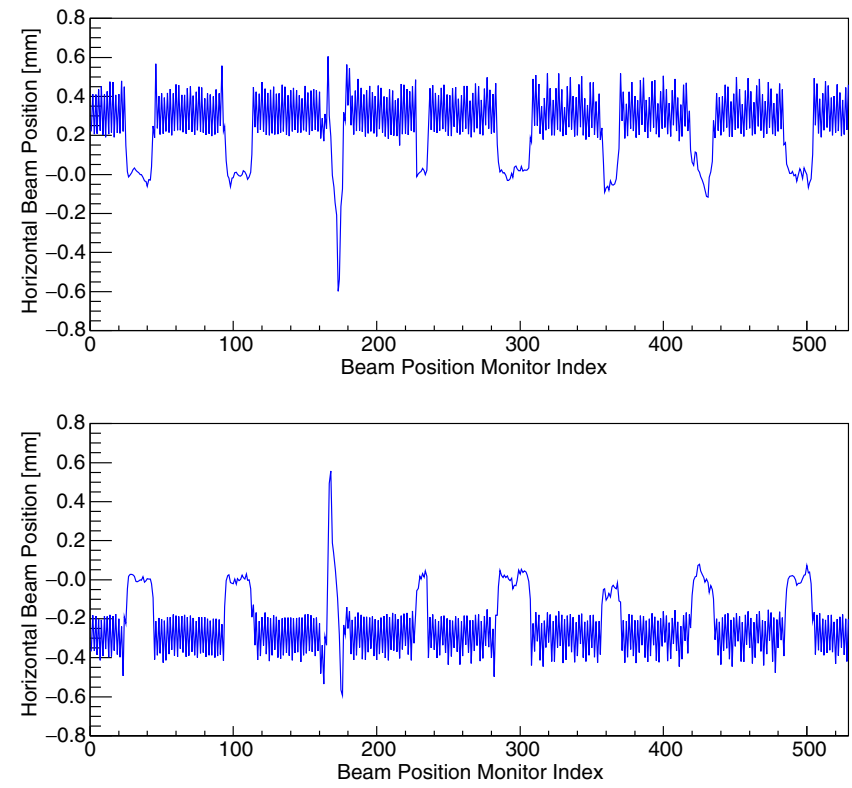

FIG. 10. Beam position change for the protons in ring 1 (top) and lead ions in ring 2 (bottom) between start and end of the rf manipulations at $4 \mathrm{TeV} / \mathrm{c}$. For both plots the horizontal axis represents the BPM index, while the vertical axis is the horizontal (radial) beam position measured at each BPM in $\mathrm{mm}$. The position shift is modulated by the horizontal dispersion $D_{x, i}$ at each BPM. 
displays the orbit shift of the two beams when they are forced to the common frequency during the rf manipulation at $4 \mathrm{TeV} / \mathrm{c}$. The typical mean radial change is $0.3 \mathrm{~mm}$ per beam, a $1 \mathrm{~Hz}$ frequency change shifting the beam radially by $10.6 \mu \mathrm{m}$. Systematic errors on the radial position determination due to BPM scale and offset errors are the limiting factor for the measurement accuracy.

The relative momentum offset of the beam due to its radial shift can be estimated from a least square fit to the horizontal BPM readings which yields the following equation,

$$
\frac{\Delta P}{P}=\frac{\sum_{i=1}^{N} D_{x, i} x_{i}}{\sum_{i=1}^{N} D_{x, i}^{2}}
$$

where $i$ labels the BPMs and $N$ is the total number of BPMs. $D_{x, i}$ is the horizontal dispersion at the BPM with index $i$ and $x_{i}$ is the measured horizontal beam position at the same BPM. The momentum offset is converted into a rf frequency offset with the momentum compaction factor $\alpha_{c}$

$$
\Delta f_{\mathrm{rf}}=-\frac{f_{\mathrm{rf}}}{\alpha_{c}} \frac{\Delta P}{P}=-\frac{f_{\mathrm{rf}}}{\alpha_{c}} \frac{\sum_{i=1}^{N} D_{x, i} x_{i}}{\sum_{i=1}^{N} D_{x, i}^{2}} .
$$

For the LHC $\alpha_{c}=3.2 \times 10^{-4}$.

The main issue for the accuracy of the measurements of the frequency offset is coming from possible systematic offsets between the center of the BPMs and the center of the quadrupoles. A mean systematic alignment offset will lead to an error on the reconstructed frequency offset, limiting the accuracy when for example a single configuration with protons in ring 1 and lead ions in ring 2 is used. For a constant measurement offset of the BPMs, it is possible to cancel the systematic error by inverting the beams in the rings, i.e. by measuring in the configuration with protons in ring 1 and lead ions in ring $2(\mathrm{pPb})$ as well as in the reverse configuration with protons in ring 2 and lead ions in ring $1(\mathrm{Pbp})$.

If $\delta_{f 1}\left(\delta_{f 2}\right)$ is the systematic frequency error that is introduced by a center offset of the BPMs in ring 1 (ring 2), see Fig. 11, the measured frequency difference $\Delta f_{p P b}$ between proton and lead in $\mathrm{pPb}$ configuration is given by

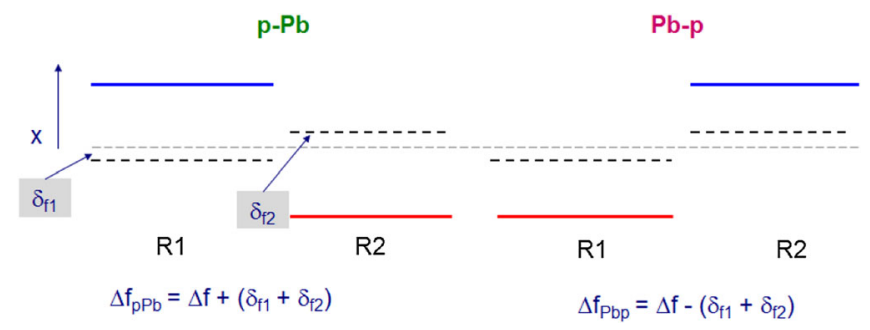

FIG. 11. Principle of the cancellation of systematic errors on the radial position by inversion of the protons and lead ions in the two rings.

$$
\Delta f_{p P b}=\Delta f_{\mathrm{rf}}+\left(\delta_{f 1}+\delta_{f 2}\right),
$$

while in Pbp configuration the measurement yields

$$
\Delta f_{P b p}=\Delta f_{\mathrm{rf}}-\left(\delta_{f 1}+\delta_{f 2}\right) .
$$

$\Delta f_{\text {rf }}$ represents the true frequency offset between protons and lead ions. It is assumed that the BPMs are only sensitive to the beam charge and do not change their characteristics when the beams are inverted. The real frequency is obtained by averaging the measurements taken in the two configurations since the systematic errors have exactly the opposite sign

$$
\Delta f_{\mathrm{rf}}=\frac{1}{2}\left(\Delta f_{p P b}+\Delta f_{P b p}\right)
$$

The potential of removing or at least reducing drastically the systematic error from center offsets of BPMs and quadrupoles makes the mixed mode with proton and lead ions attractive for the energy measurement.

\section{ENERGY MEASUREMENTS WITH PROTON-LEAD BEAMS}

The frequency difference between proton and lead beams is reconstructed with two methods. The first method (A) determines the difference before beam cogging, when both beams are approximately centered radially and operated with different $\mathrm{rf}$ frequencies. In that case the dominant part of the frequency difference is encoded in the $\mathrm{rf}$ frequencies of the two rings. A correction must be applied to account for possible radial offsets of the two beams with respect to the mean center of the BPMs. The second method (B) determines the frequency difference after cogging when both beams are operated at the same rf frequency, but offset radially, see Fig. 10. The systematic errors are different for the two methods. For method A for example the scale of the BPMs is less important than for method B. Systematic offsets of the BPMs play however a similar role in both cases, since the frequency differences of the beams are in both cases obtained with respect to the centered BPM readings.

The measurements presented in this section were obtained parasitically to regular machine operation. No machine time could be dedicated to special studies due to the short length of the proton lead runs and the extremely dense experimental program. The results presented for the 2013 run at $4 \mathrm{TeV} / \mathrm{c}$ and $450 \mathrm{GeV} / \mathrm{c}$ are revised results of an already published CERN report [29].

\section{A. Energy measurement at $4 \mathrm{TeV} / \mathrm{c}$}

Since the frequency shifts are obtained from the radial beam position measured by BPMs the calibration of the BPM scale is important to obtain accurate measurements, in particular after rf cogging. The BPM scale correction 

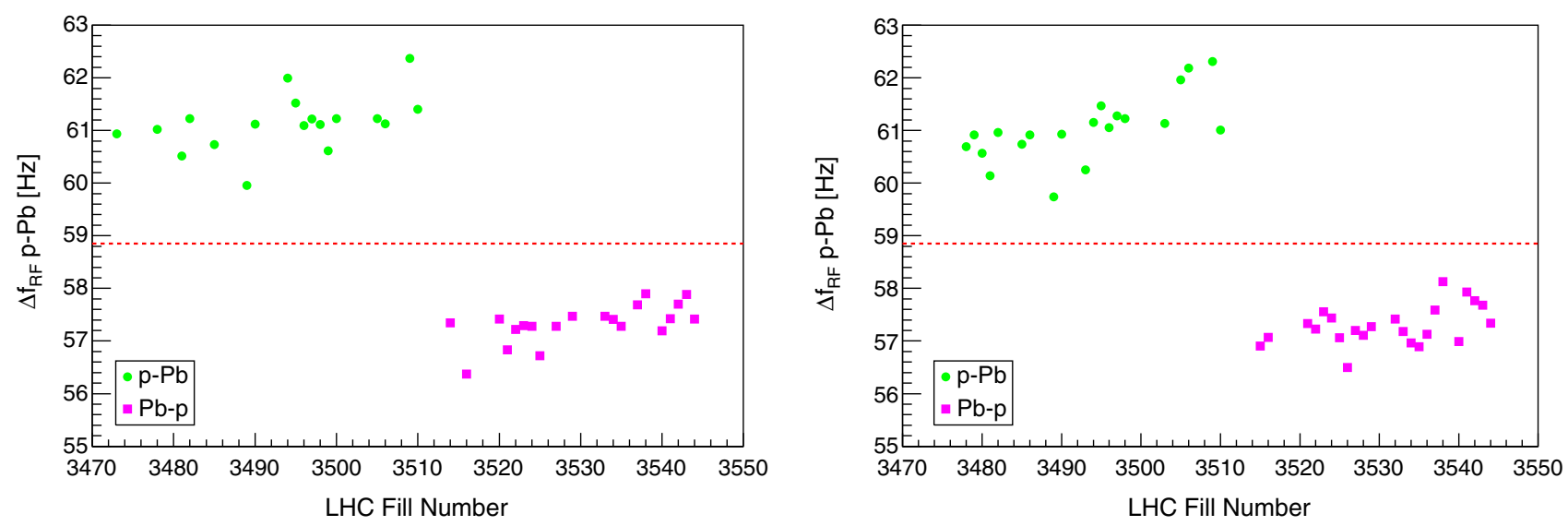

FIG. 12. Time dependence of the frequency difference $\Delta f_{\text {rf }}$ of protons and lead ions at $4 \mathrm{TeV} / \mathrm{c}$ as a function of the LHC fill number in 2013. For the figure on the left, the frequency is reconstructed after the rf cogging (method B), for the figure on the right before rf cogging (method A). The red dotted line is the frequency difference corresponding to an energy of $4 \mathrm{TeV} / \mathrm{c}$.

was determined at $4 \mathrm{TeV} / \mathrm{c}$ with controlled rf frequency trims of $10 \mathrm{~Hz}$ over a range of $\pm 40 \mathrm{~Hz}$ around the central orbit. The calibrations were performed in 3 fills during the proton-lead operation period. In all cases the response of the BPMs was perfectly linear. The calibrations were reproducible and yielded a BPM scale correction factor of $C_{1}=1.020 \pm 0.001$ for ring 1 and $C_{2}=1.023 \pm 0.003$ for ring 2. $C_{1(2)}$ are the scale corrections to be applied to the BPM estimate based on the ideal machine dispersion, they correspond to the ratio of frequency change reconstructed by the BPMs with respect to the true frequency change. The fact that $C_{1(2)}$ are larger than 1 implies that the BPMs overestimate the radial orbit offsets. The calibration of the BPM scale with respect to the rf frequency provides an accurate normalization without need to measure the dispersion at each BPM with high accuracy; only the convolution of BPM scale and dispersion must be accurately calibrated.

The bunch current is important for the orbit reconstruction because of a dependance of the BPM electronics accuracy on this beam parameter. The sensitivity is due to the principle of the wide band time normalization electronics [30]. To assess the influence on the measurement of the radial orbits and reconstructed frequencies, bunches were scraped on collimators at injection during stable conditions. The apparent change of the radial orbit position due to intensity systematics was then assessed. For ring 1 the radial position measurement was stable down to $\approx 6 \times 10^{9}$ electron charges per bunch, while for ring 2 systematic effects become visible around $\approx 10^{10}$ electron charges. This difference is explained by the presence of (unused) intensity measurement electronics in the ring 1 BPM acquisition chain, affecting the electronics systematic errors in 2013. This intensity measurement electronics was removed in 2014 and does not affect the 2016 data. While this systematic effect does not impact the measurement of the protons due to the higher bunch charge, the lead ion bunch measurements in $\mathrm{pPb}$ configuration with lead in ring 2 are in a region where the systematic errors may reach $0.5 \mathrm{~Hz}$ according to the scraping tests. This will be considered later for the systematic errors.

The frequency difference between protons and lead ions is extracted from orbits acquired at a standard time corresponding to the end of the ramp for method A (before frequency cogging) and to the end of the cogging for method B (Sec. VII B). For each beam the frequency offset with respect to the center of the BPMs is determined using Eq. (23). For method A the measured rf frequency differences are corrected for residual radial offsets. For method B the frequencies are reconstructed from the orbit differences. In both cases the orbit shifts are corrected for the BPM scale calibration factors $C_{1(2)}$.

The reconstructed frequency difference $\Delta f_{\mathrm{rf}}$ is shown for all fills where the lead beam intensity was above $6 \times 10^{9}$ charges per bunch in Fig. 12. The results of the two methods agree within $0.1 \mathrm{~Hz}$, consistent with the statistical uncertainties. The offset of $\approx 4 \mathrm{~Hz}$ between the data for the two beam directions reflects the systematic error on the frequency offset described in Sec. VII C. The $4 \mathrm{~Hz}$ difference is equivalent to a radial offset $\simeq 40 \mu \mathrm{m}$. To obtain an accurate result the data of the two periods must be averaged.

To evaluate systematic effects the data was analysed using different cuts and subsamples of the BPMs. Different BPM samples were considered by selecting only BPMs with large horizontal dispersion, selecting only BPMs in half of the ring or varying the cuts on bunch length or bunch intensity. A systematic error of $\pm 0.25 \mathrm{~Hz}$ is assigned to the choice of BPM selection. Since the lead ion bunch intensities lie in a range where BPM systematic error have a small impact, a systematic error of $\pm 0.25 \mathrm{~Hz}$ was assigned to this effect. A systematic error of $0.5 \%$ is assigned to the scale calibration, which results in a $0.3 \mathrm{~Hz}$ systematic error on the frequency for method B, and less than $0.1 \mathrm{~Hz}$ for method $\mathrm{A}$. The total systematic 

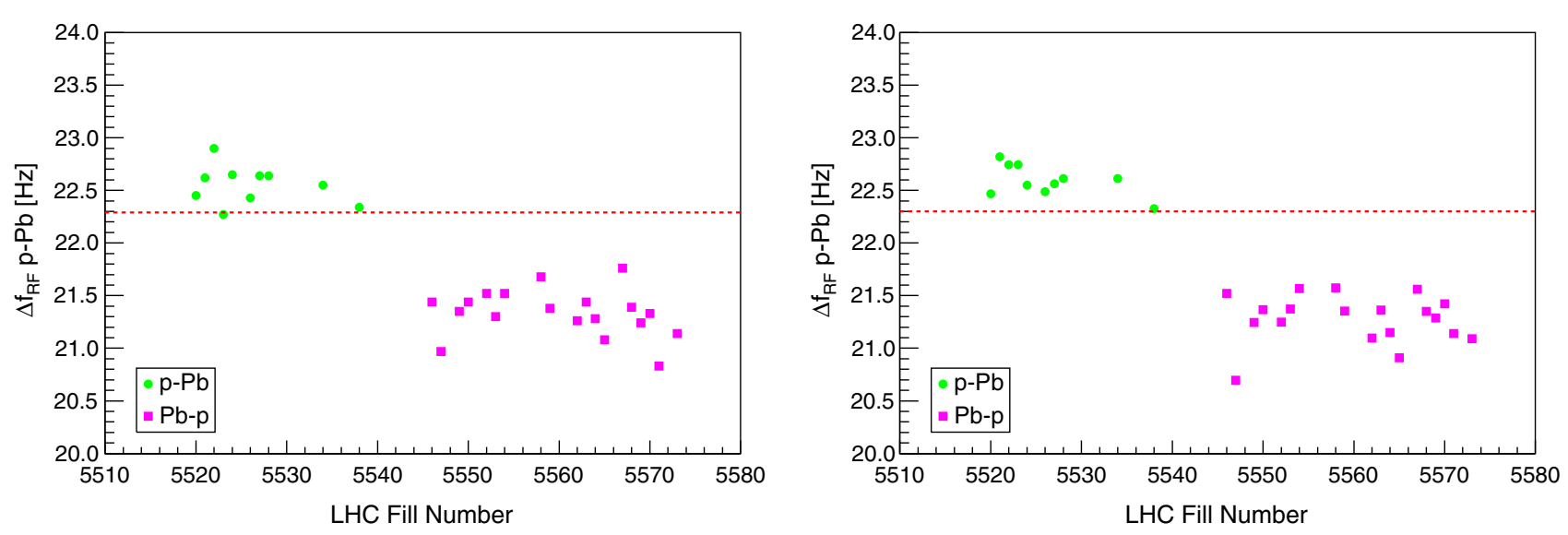

FIG. 13. Time dependence of the frequency difference $\Delta f_{\text {rf }}$ of protons and lead ions at $6.5 \mathrm{TeV} / \mathrm{c}$ as a function of the LHC fill number in 2016. For the figure on the left, the frequency is reconstructed after the rf cogging (method B), for the figure on the right before $\mathrm{rf}$ cogging (method A). The red dotted line is the frequency difference corresponding to an energy of $6.5 \mathrm{TeV} / \mathrm{c}$.

error is obtained from the quadratic sum of all contributions, yielding a final error of $\pm 0.4 \mathrm{~Hz}$ for method $\mathrm{A}$. The result for the frequency difference between protons and lead ions at $4 \mathrm{TeV} / \mathrm{c}$ for method $\mathrm{A}$, which has slightly smaller systematic errors, is

$$
\Delta f_{\mathrm{rf}, 4 \mathrm{TeV}}=59.16 \pm 0.16 \text { (stat) } \pm 0.4 \text { (syst) Hz. }
$$

This result can be converted into a beam momentum of

$$
\begin{aligned}
P_{4 \mathrm{TeV}} & =3990 \pm 5(\text { stat }) \pm 14(\text { syst }) \mathrm{GeV} / \mathrm{c} \\
& =3990 \pm 15 \mathrm{GeV} / \mathrm{c}
\end{aligned}
$$

where the total error is obtained from the quadratic sum of the statistical and of the systematic error. The value is in excellent agreement with the $4 \mathrm{TeV} / \mathrm{c}$ expected from the magnetic model.

\section{B. Energy measurement at $6.5 \mathrm{TeV} / \mathrm{c}$}

The frequency difference of proton and lead beams was measured at $6.5 \mathrm{TeV} / \mathrm{c}$ during the $2016 \mathrm{LHC}$ run. The frequency measurement was again performed at top energy before beam cogging, with both beams approximately centered at different $\mathrm{rf}$ frequencies (method A). The measurement was repeated after cogging with both beams at the same rf frequency, but offset radially (method B). The scale of the BPMs was determined to be $1.025 \pm 0.004$, consistent with the 2013 run results at $4 \mathrm{TeV} / \mathrm{c}$. The results for both methods are presented in Fig. 13 for all fills where the beams consisted of trains with $100 \mathrm{~ns}$ bunch spacing. The two methods again yield consistent results. The systematic shift between $\mathrm{pPb}$ and $\mathrm{Pbp}$ configurations has the same trend than at $4 \mathrm{TeV} / \mathrm{c}$, but with a smaller offset, possibly due to the modifications to machine and electronics during the long shutdown in 2013 and 2014. The overall BPM quality and reproducibility was improved significantly with better cooling and temperature regulation of the acquisition electronics.

The systematic error assigned to the BPM selection is $\pm 0.25 \mathrm{~Hz}$, identical to the $4 \mathrm{TeV} / \mathrm{c}$ value. The effect of a scale uncertainty of $0.5 \%$ is only $0.05 \mathrm{~Hz}$ due to the small radial offsets. Due to the higher ion bunch intensity, the effect of the intensity does not exceed $\pm 0.12 \mathrm{~Hz}$. The total systematic error is estimated to $\pm 0.28 \mathrm{~Hz}$.

The results of the two measurement methods agree with an average frequency of

$\Delta f_{\mathrm{rf}, 6.5 \mathrm{TeV}}=21.95 \pm 0.12$ (stat) \pm 0.28 (syst) Hz.

This results in an energy of

$$
\begin{aligned}
P_{6.5 \mathrm{TeV}} & =6551 \pm 17(\text { stat }) \pm 41(\text { syst }) \mathrm{GeV} / \mathrm{c} \\
& =6551 \pm 45 \mathrm{GeV} / \mathrm{c} .
\end{aligned}
$$

This value is consistent with the magnetic model of the LHC, but the uncertainty of $0.7 \%$ is again significantly larger than the uncertainty on the LHC magnetic model.

\section{Energy measurement at injection}

The injection energy was measured with proton and lead beams in 2013 and 2016. At that moment of the cycle the rf systems of the two rings are still uncoupled as described in Sec. VII B and the beams are operated with a large rf frequency difference. The frequency offset between protons and lead ions is obtained from the recorded rf frequencies for each ring, corrected for the radial offset measured by the BPMs which corresponds to method A described in the previous sections. In general the beams are well centered in this phase, and the corrections from the orbit measurements are small, typically less than $\pm 10 \mathrm{~Hz}$, compared to the total frequency difference of $4.6 \mathrm{kHz}$. The BPM scale calibration uncertainty at injection is therefore estimated to be $0.5 \mathrm{~Hz}$. 

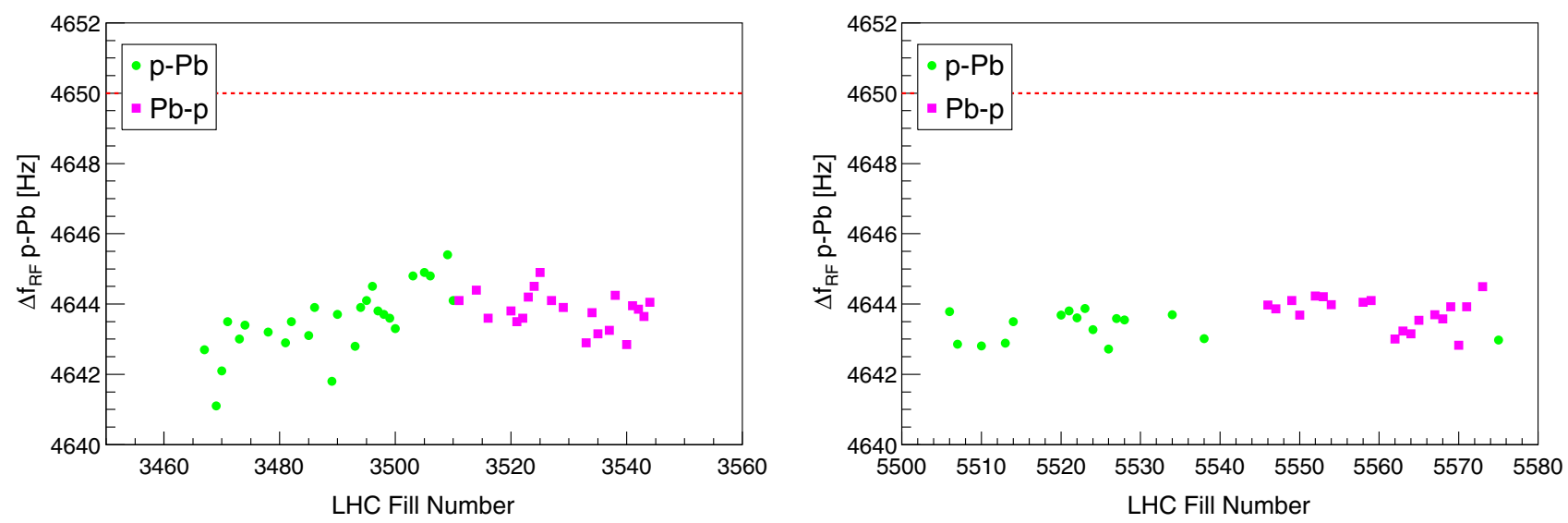

FIG. 14. Time dependence of the frequency difference $\Delta f_{\text {rf }}$ of protons and lead ions as a function of the LHC fill number in 2013 (left) and 2016 (right). The red dotted line is the frequency difference corresponding to an energy of $450.0 \mathrm{GeV} / \mathrm{c}$.

Figure 14 presents the evolution of the proton and lead ion $\mathrm{rf}$ frequency difference as a function of the fill number at $450 \mathrm{GeV}$ for the two runs. In each period the orbits and corrector settings were stable or were compensated. Contrary to the situation at higher energy, the offset of the measurements between the $\mathrm{pPb}$ and $\mathrm{Pbp}$ periods is small. This difference can be explained by the absolute orbit at injection which differs from the orbit at high energy. In addition the corrector settings are not identical which also influences the mean position of the beams in the BPMs of the two rings.

Assuming conservatively a total systematic error of $\pm 1 \mathrm{~Hz}$ on the averaged frequency difference at injection, the result for the frequency difference between protons and lead ions at $450 \mathrm{GeV}$ for 2013 is

$$
\Delta f_{\text {rf,inj }}=4643.7 \pm 0.2(\text { stat }) \pm 1.0(\text { syst }) \mathrm{Hz}
$$

while for 2016 the value is

$$
\Delta f_{\text {rf,inj }}=4643.6 \pm 0.1 \text { (stat) } \pm 1.0 \text { (syst) Hz. }
$$

The values of both runs are in excellent agreement which is also visible in Fig. 14. The systematic error of $1 \mathrm{~Hz}$ is considered conservatively to be fully correlated between the two runs.

The combined result can be converted into a beam momentum at injection of

$$
P_{\text {inj }}=450.31 \pm 0.01 \text { (stat) } \pm 0.07 \text { (syst) GeV/c. }
$$

A contribution to the systematic error from the orbit corrector magnets of $0.05 \mathrm{GeV}$ was added in quadrature to the uncertainty arising from the frequency difference. This contribution is not negligible at injection due to the much smaller relative uncertainty on the beam energy.

\section{SUMMARY}

Table IV presents a summary of the errors on the LHC beam energy above injection energy. The relative error is $0.1 \%$. As discussed in the previous sections of this document, the uncertainty on the transfer function dominates all other contributions. The energy values do not require corrections, i.e. the nominal values as reconstructed and distributed online to the experiments are applicable.

At injection energy the relative uncertainty on the beam energy is only $0.024 \%$ and the central value is shifted with respect to the nominal energy setting of $450 \mathrm{GeV} / \mathrm{c}$ by $+0.31 \mathrm{GeV} / \mathrm{c}$. The measured energy at injection is in excellent agreement with the magnetic model and its estimated uncertainty. Table $\mathrm{V}$ presents a summary of the energies and their uncertainties.

The uncertainties of the energies for ring 1 and ring 2 are fully correlated.

For mixed proton and lead operation the energies of the two beams differ systematically during physics data taking, by $0.046 \%$ at $4 \mathrm{TeV} / \mathrm{c}$ and by $0.018 \%$ at $6.5 \mathrm{TeV} / \mathrm{c}$. Due to the antisymmetry of the energy shifts (upwards for protons, downward for lead ions) the center of mass energy is not affected by this effect.

The direct measurement of the beam energy using proton and lead ion provide an accurate calibration at injection where the accuracy of the magnetic model is also confirmed. The direct measurements at $4 \mathrm{TeV} / \mathrm{c}$ and $6.5 \mathrm{TeV} / \mathrm{c}$

TABLE IV. Table of relative errors to the LHC beam energy above injection energy.

\begin{tabular}{lc}
\hline \hline Contribution & Error $(\%)$ \\
\hline PC calibration & 0.001 \\
Slow radial changes & 0.005 \\
Earth tides & 0.005 \\
Orbit correctors & 0.03 \\
Transfer function & 0.1 \\
Sum & 0.1 \\
\hline \hline
\end{tabular}


TABLE V. Reference (online) energies, actual energies and their uncertainties are given for all energy settings that have been used for LHC operation since 2010.

\begin{tabular}{lcc}
\hline \hline $\begin{array}{l}\text { Nominal momentum } \\
(\mathrm{GeV} / \mathrm{c})\end{array}$ & $\begin{array}{c}\text { Actual momentum } \\
(\mathrm{GeV} / \mathrm{c})\end{array}$ & $\begin{array}{c}\text { Relative } \\
\text { uncertainty }(\%)\end{array}$ \\
\hline 450 & 450.31 & 0.024 \\
1380 & 1380 & 0.1 \\
2510 & 2510 & 0.1 \\
3500 & 3500 & 0.1 \\
4000 & 4000 & 0.1 \\
6370 & 6370 & 0.1 \\
6500 & 6500 & 0.1 \\
\hline \hline
\end{tabular}

are in good agreement with the magnetic model, but the accuracy is roughly a factor four to seven worse than the estimated error of the magnetic model.

\section{ACKNOWLEDGMENTS}

We would like to acknowledge the efficient and smooth collaboration with John Jowett during the proton lead runs. We would also like to thank the LHC physics coordinators Jamie Boyd and Christoph Schwick for the continuous support and encouragement to prepare this publication.

[1] P. Grafstrom and W. Kozanecki, Luminosity determination at proton colliders, Prog. Part. Nucl. Phys. 81, 97 (2015).

[2] M. Aaboud et al. (ATLAS Collaboration), Luminosity determination in pp collisions at $\sqrt{s}=8 \mathrm{TeV}$ using the ATLAS detector at the LHC, Eur. Phys. J. C 76, 653 (2016).

[3] V. Khachatryan et al. (CMS Collaboration), Report No. CMS-PAS-LUM 13-001, 2013.

[4] R. Aaij et al. (LHCb Collaboration), Precision luminosity measurements at LHCb, J. Instrum. 9, P12005 (2014).

[5] J. Adam et al. (ALICE Collaboration), Report No. ALICEPUBLIC 2016-002, 2016.

[6] G. Antchev et al. (TOTEM Collaboration), LuminosityIndependent Measurement of the Proton-Proton Total Cross Section at $\sqrt{s}=8 \mathrm{TeV}$, Phys. Rev. Lett. 111, 012001 (2013).

[7] M. Aaboud et al. (ATLAS Collaboration), Measurement of the total cross section from elastic scattering in pp collisions at $\sqrt{s}=8 \mathrm{TeV}$ with the ATLAS detector, Phys. Lett. B 761, 158 (2016).

[8] R. Assmann et al., Eur. Phys. J. C 6, 187 (1999).
[9] The LEP Energy Working Group, Calibration of centre-ofmass energies at LEP 2 for a precise measurement of the W boson mass, Eur. Phys. J. C 39, 253 (2005).

[10] J. Wenninger, LHC Project Report No. 334, 2004.

[11] L. Rossi, The LHC main dipoles and quadrupoles toward series production, IEEE Trans. Appl. Supercond. 13, 1221 (2012).

[12] A. Verweij et al., in Proceedings of the 11th European Particle Accelerator Conference, Genoa, 2008 (EPS-AG, Genoa, Italy, 2008).

[13] G. Fernqvist, The measurement challenge of the LHC project, IEEE Trans. Instrum. Meas. 48, 9000104 (1999).

[14] N. Aquilina, M. Lamont, M. Strzeclzyk, E. Todesco, and N. Sammut, Chromaticity decay due to superconducting dipoles on the injection plateau of the Large Hadron Collider, Phys. Rev. ST Accel. Beams 15, 032401 (2012).

[15] N. Aquilina, M. Giovannozzi, M. Lamont, N. Sammut, R. Steinhagen, E. Todesco, and J. Wenninger, Tune variations in the Large Hadron Collider, Nucl. Instrum. Methods Phys. Res., Sect. A 778, 6 (2015).

[16] A. Jain, CERN yellow Report No. 98-05, p. 175, 1998.

[17] L. Bottura et al., LHC Project Report No. 326, 2004.

[18] S. Russenschuck and B. Auchmann, Technical Report, CERN, 2007.

[19] P. Hagen, B. Auchmann, and E. Todesco, Comparison between electromagnetic models and magnetic measurements in the LHC magnets, IEEE Trans. Appl. Supercond. 22, 9000104 (2012).

[20] L. Arnaudon et al., Effects of terrestrial tides on the LEP beam energy, Nucl. Instrum. Methods Phys. Res., Sect. A 357, 249 (1995).

[21] J. Wenninger, in Proceedings of the 18th Particle Accelerator Conference, New York, 1999 (IEEE, New York, 1999).

[22] J. Wenninger, Report No. SL-Note 2000-09 OP, 2000.

[23] E. Todesco et al., in Proceedings of the 3rd International Particle Accelerator Conference, New Orleans, LA, 2012 (IEEE, Piscataway, NJ, 2012).

[24] R. Bailey et al., in Proceedings of EPAC90, European Particle Accelerator Conference (Ed. Frontieres, Gif-surYvette, 1990).

[25] X. Altuna et al., Report No. CERN SL 92-32, 1992.

[26] G. Arduini et al., Report No. AB-Note 2003-014 OP, 2003.

[27] J. Jowett et al., CERN Report No. CERN-ATS-Note 2012$052 \mathrm{MD}, 2012$.

[28] J. Jowett et al., CERN Report No. CERN-ATS-Note 2012094 MD, 2012.

[29] J. Wenninger, CERN Report No. CERN-ATS 2013-040, 2013.

[30] E. Calvo-Giraldo et al., in Proceedings of the 6th DIPAC, European Workshop on Beam Diagnostics and Instrumentation for Particle Accelerators (Jacow, Darmstadt, 2003). 